\title{
APPROXIMATE ANALYTICAL DESCRIPTION OF THE UNDERDENSE SHORT PLASMA LENS
}

\section{A.Ts. Amatuni}

Yerevan Physics Institute

\author{
Alikhanian Brother's St. 2, Yerevan 375036, Republic of Armenia
}

\begin{abstract}
The perturbative approach for describing the underdense plasma-ultrarelativistic electron bunch system is developed,using the ratio $\frac{n_{0}}{n_{b}}$ as a small parameter $\left(n_{b^{-}}\right.$-bunch, $n_{0^{-}}$ plasma electron densities). Focusing of the electron bunch emerged in the first approximation of the perturbative procedure as a result of the plasma electrons redistribution.Focusing gradient and strength for ultrarelativistic,flat,uniform and short bunch are obtained and compared with the previous results.

keywords:plasma focusing,underdense plasma lens,perturbative approach

\section{INTRODUCTION}

Plasma focusing devices, being compact,simple and effective elements are promissing for obtaining electron (positron) beams of very small spot size, requested for future high energy linear colliders. Theoretical predictions and investigations of the plasma lenses in overdense $\left(n_{b}<n_{0} \equiv n_{p}\right)$ and underdense $\left(n_{b}>n_{0}, n_{b}\right.$-bunch electron density, $n_{0}$-cold neitral plasma electron density),performed last decade [1]-8] and were supplemented by experimental tests,carried out at ANL [9]-10], Tokio University-KEK [11] and UCLA [12] for overdense plasma lens regime.

The theoretical treatment of the overdense plasma lens,performed in linear approximation, using ratio $\frac{n_{b}}{n_{0}}$ as a small parameter, is more or less complete, at least in the frame of the cold plasma and rigid electron bunch approximations.

The existing theoretical approaches to underdense plasma lens are phenomenological by nature.
\end{abstract}


It seems,that more detailed description of the underdense plasma lens is needed,in particular, taking into account the program for the future experimental investigations [13], which include underdense regime too.

Using the ratio $\frac{n_{0}}{n_{b}} \ll 1$ as a small parameter,and developing the subsequent perturbative approach for the description of the underdense plasma lens,it is possible to achieve, at least the same level of the understanding of the focusing phenomenon in underdense regime as that of overdense plasma lens case.

The present work is devoted to this goal.In the next section the perturbative approach is developed for underdense plasma regime for the system of the Maxwell equations and hydrodinamical equations of the motion of the plasma electrons (ions are assumed immobile).The third section devoted to zero approximation calculations of the generated fields and plasma electron velocities, assuming that bunch is ultrarelativistic and short enough.In the section 4 the first order approximation results for plasma electrons redistri-

bution and transverse force (sum of the electric and magnetic forces) are given.Section 5 contents the disscusion of the obtained results.In particular,it is mentioned that physical picture of focusing phenomenon in underdense case is based on the redistribution of the plasma electrons around the driving bunch. The effect of the ion column in ultrarelativistic case is negligible,compared to the focusing due to redistributed plasma electrons.

The mathematical technique developed and above mentioned physical discription of the focusing phenomenon may be used for more detailed,coherent and associated with the experimental program [13] computer calculations.

\section{PERTURBATIVE APPROACH FOR UNDERDENSE PLASMA LENS}

For definiteness, consider the flat electron bunch with the vertical dimension $2 b$, which is assumed much smaller than horizontal dimension $2 a$; longitudinal dimensions, which are arbitrary,are $2 d$.The bunches of such a geometry are suitable for future high energy linear electron-positron colliders [13].

Bunch electrons uniform density is $n_{b}$ and bunch is considered as ultrarelativistic 
and rigid one.Plasma electrons density is $n_{0} \ll n_{b}$,plasma is neutral,cold and with the immobile ions.

The geometry of the bunch,which moves in lab system through the plasma with constant velocity $v_{0}$,allows to consider the electric and magnetic fields components as follows:

$$
E_{x}=0, E_{y}, E_{z} \neq 0 ; B_{x} \neq 0, B_{y}=B_{z}=0
$$

This more or less evident approximation for the flat beams with large aspect ratio,along with the condition $\frac{\partial}{\partial x} \ll \frac{\partial}{\partial y}$ was introduced in [14].In [15] it was pointed out, that the assumption $B_{y} \ll B_{x} \equiv B$ "is not universally true everywhere inside the even very flat beam". It is neccessary to take this into account at precise analytical or computer calculations.

All physical quantities of the problem are considered as a functions of the arguments $y$ and $\tilde{z}=z-v_{0} t$ only (steady state regime). Introduce the dimensionless arguments $y_{1}^{\prime} \tilde{z}^{\prime}=k_{b} y, k_{b} \tilde{z} ; t^{\prime}=\omega_{b} t, \omega_{b}^{2}=\frac{4 \pi e^{2} n_{b}}{m}, k_{b}=\frac{\omega_{b}}{c}$, and dimensionless variables $E_{y, z}^{\prime}=\left(\frac{\omega_{b} m c}{e}\right)^{-1} E_{y, z} ; B^{\prime}=\left(\frac{\omega_{b} m c}{e}\right)^{-1} B_{x}$;

Followig [16, 17, 8]define the generalized plasma electron velocities as

$V_{y}=\frac{v_{e y}}{v_{0}-v_{e z}}=\frac{\beta_{e y}}{\beta-\beta_{e z}}, \beta=\frac{v_{0}}{c}, \beta_{e z, y}=\frac{v_{e, z y}}{c}, V_{z}=\frac{v_{e z}}{v_{0}-v_{e z}}=\frac{\beta_{e z}}{\beta-\beta_{e z}}$

and generalized plasma electron density as

$N=\frac{n_{e}}{n_{b}}\left(1-\frac{v_{e z}}{v_{0}}\right)=\frac{n_{e}}{n_{b}}\left(1-\frac{\beta_{e z}}{\beta}\right), \frac{n_{e}}{n_{b}}=\frac{\beta N}{\beta-\beta_{e z}}=N\left(1+V_{z}\right)$,

(BTFCh transformations [16]), it is possible to rewrite the continuity equation in the following form:

$$
-\frac{\partial N}{\partial z}+\frac{\partial\left(N V_{y}\right)}{\partial y}=0
$$

(in (11) and what follows the tilda and prime superscripts are removed). The Maxwell equations in this case are: 


$$
\begin{array}{ll}
\text { 1. } & \frac{\partial B}{\partial y}=\beta+\beta N V_{z}+\beta \frac{\partial E}{\partial z}, \\
\text { 2. } & \frac{\partial}{\partial z}\left(B+\beta E_{y}\right)=-\beta N V_{y}, \\
\text { 3. } & \frac{\partial}{\partial z}\left(\beta B+E_{y}\right)=\frac{\partial E_{z}}{\partial y}, \\
\text { 4. } & \frac{\partial E_{z}}{\partial z}+\frac{\partial E_{y}}{\partial y}=-\left[1-n_{0} / n_{b}+N\left(1+V_{z}\right)\right],
\end{array}
$$

Eqs. (2) are valid for $-d \leq z \leq d,-b \leq y \leq b$;when $|z|>d,|y|>b$ in right hand side of eqs. (2.1) and (2.4) the quantities $\beta$ and -1 subsequently are absent.Eq. (2.4) follows from (11), (2.1), (2).2).

The eqs. of the motion are:

$$
\begin{aligned}
& \text { 1. } \quad \dot{V}_{z}=\frac{V^{1 / 2}}{\beta^{2}}\left[-E_{z}\left(1+2 V_{z}+\frac{V_{z}^{2}}{\gamma^{2}}\right)+\beta^{2} V_{y} V_{z}\left(E_{y}+\frac{1}{\beta} B\right)+\beta V_{y} B\right], \\
& \text { 2. } \dot{V}_{y}=\frac{V^{1 / 2}}{\beta^{2}}\left[-E_{y}\left(1+V_{z}-\beta^{2} V_{y}^{2}\right)-E_{z} V_{y}\left(1+\frac{V_{z}}{\gamma^{2}}\right)-\beta\left(V_{z}-V_{y}^{2}\right) B\right]
\end{aligned}
$$

where

$$
\begin{gathered}
V \equiv 1+2 V_{z}+\frac{V_{z}^{2}}{\gamma^{2}}-\beta^{2} V_{z}^{2} \\
\dot{V}_{y} \equiv \frac{\partial V_{y}}{\partial z}+V_{y} \frac{\partial V_{y}}{\partial y}, \dot{V}_{z} \equiv-\frac{\partial V_{z}}{\partial z}+V_{y} \frac{\partial V_{z}}{\partial y} ;
\end{gathered}
$$

Adopting the condition $\frac{n_{o}}{n_{b}} \ll 1$,decompose the quantities in question in the following series:

$$
\begin{array}{r}
N=\epsilon N_{1}+\epsilon^{2} N_{2}+\ldots \\
V_{z}=V_{z 0}+\epsilon V_{z 1}+\epsilon^{2} V_{z 2}+\ldots \\
V_{y}=V_{y 0}+\epsilon V_{y 1}+\epsilon^{2} V_{y 2}+\ldots \\
E_{y, z}=E_{y, z 0}+\epsilon E_{y, z 1}+\epsilon^{2} E_{y, z 2}+\ldots \\
B=B_{0}+\epsilon B_{1}+\epsilon^{2} B_{2}+\ldots
\end{array}
$$


where $\epsilon=\frac{n_{0}}{n_{b}}$ (in what follows $\epsilon^{n}$ is included in subsequent quantities $N_{n}, V_{n z}$ and so on)

In the zero order approximation from (2.2) and (2.3)

$$
B_{0}=-\beta E_{y 0}, \frac{1}{\gamma^{2}} \frac{\partial E_{y 0}}{\partial z}=\frac{\partial E_{z 0}}{\partial y}
$$

From (7) and (2.4),introducing the potential $\varphi_{0}(z, y)$ by

$$
E_{z 0}=-\frac{1}{\gamma^{2}} \frac{\partial \varphi_{0}}{\partial z}, E_{y 0}=-\frac{\partial \varphi_{0}}{\partial y}
$$

it follows that

$$
\frac{1}{\gamma^{2}} \frac{\partial^{2} \varphi_{0}}{\partial z^{2}}+\frac{\partial^{2} \varphi_{0}}{\partial y^{2}}=\left\{\begin{array}{l}
1, \quad-d \leq z \leq d, \quad-1 \leq y \leq b \\
0, \quad|z|>d,|y|<b
\end{array}\right.
$$

In the first order approximation:

$$
-\frac{\partial N_{1}}{\partial z}+\frac{\partial N_{1} V_{y 0}}{\partial y}=0
$$

from (10) and (2) follows:

$$
\frac{\partial^{2} E_{z 1}}{\partial z^{2}}+\gamma^{2} \frac{\partial^{2} E_{z 1}}{\partial y^{2}}=\frac{\partial}{\partial z}\left(\frac{n_{0}}{n_{b}}-\gamma^{2} N_{1}-N_{1} V_{z 0}\right)
$$

The focusing force is

$$
\begin{gathered}
f_{y}=-e\left(E_{y}+\beta B\right)=f_{y 0}+f_{y 1}+\ldots, \\
f_{y 0}=-e\left(E_{y 0}+\beta B_{0}\right)=-e \gamma^{-2} E_{y 0} \\
f_{y 1}=-e\left(E_{y 1}+\beta B_{1}\right) \equiv-e W_{y 1} .
\end{gathered}
$$

From (2.3) in the first approximation

$$
\frac{\partial W_{y 1}}{\partial \tilde{z}}=\frac{\partial E_{z 1}}{\partial y}
$$


which is an analog of the Panoffsky-Wenzel relation;remember that in (13) $z=z-v_{0} t$. Differentating (11) over $y$ and then integrating it over $\tilde{z}$, taking into account relation (13) and that

$$
\begin{aligned}
\frac{\partial}{\partial y}\left(\frac{n_{0}}{n_{b}}-\gamma^{2} N_{1}-N_{1} V_{z 0}\right) \rightarrow 0 \text { when } y \rightarrow \pm \infty, \text { we have } \\
\qquad \frac{\partial^{2} W_{y 1}}{\partial \bar{z}^{2}}+\frac{\partial^{2} W_{y 1}}{\partial y^{2}}=\frac{1}{\gamma^{2}} \frac{\partial}{\partial y}\left(\frac{n_{0}}{n_{b}}-\gamma^{2} N_{1}-N_{1} V_{z 0}\right)
\end{aligned}
$$

where $\bar{z} \equiv \gamma \tilde{z}$.

In what follow,we consider the ultrarelativistic bunches, when $\gamma \gg 1$; then $f_{y 0} \sim$

$O\left(\gamma^{-2}\right)$ and in right hand side of (14) it is possible to leave only the term $-\frac{\partial N_{1}}{\partial y}$. So for the sought quantity $W_{y 1}$ we need $N_{1}$ from eq. (10). Entered in (10) $V_{y 0}$ must be found from eqs. (3), (9) for the fields in zero approximation.It is evident,from eq. (14), that the role of the noncompensated positiv ions (the term on the right hand side of eq. (14), proportional to $\left.\frac{n_{0}}{n_{b}}-N_{1}\left(1+V_{z 0}\right)\right)$, is negligible in ultrarelativistic limit $\left(\sim O\left(\gamma^{-2}\right)\right)$.

\section{ZERO ORDER APPROXIMATION}

In the zero order approximation it is necessary to solve eq. (9) for the flat bunch moving in vacuum $\left(\frac{n_{0}}{n_{b}}=0\right)$.

The solution may be written using Green function formalism (see e.g. 18])

$$
\varphi_{0}(\bar{z}, y)=\frac{1}{2 \pi} \int_{-\bar{d}}^{\bar{d}} d \bar{z}^{\prime} \int_{-b}^{b} d y^{\prime} \ln \left[\left(\bar{z}-\bar{z}^{\prime}\right)^{2}+\left(y-y^{\prime}\right)^{2}\right]^{1 / 2}
$$

where $\bar{d}=\gamma d$.

Double integration in (15) may be performed using standart integral tables,but the result of integration has a complex form, which is difficult to use. It is more convinient to use (8) and perform an approximate integration for $\gamma \gg 1$, taking into account, that argument of the integral has a complex pole, when $-d \leq z \leq d,-b \leq y \leq b$. Under these conditions the integration over $\bar{z}$ along the real axis in complex $\bar{z}$ plane is equal to residue 
at the pole,minus integral over the semicircle with the radii $d$ in upper complex $\bar{z}$ half plane.The approximate results of integration for $\gamma \gg 1$ are the following:

$$
\begin{gathered}
E_{y 0} \approx-y\left(1-\frac{2 b d}{\pi \gamma\left(d^{2}-z^{2}\right)}\right)=-y+O\left(\gamma^{-1}\right) \\
-b \leq y \leq b,-d \leq z \leq d ; \\
E_{y 0} \approx-b\left(1-\frac{2 d y}{\pi \gamma\left(d^{2}-z^{2}\right)}\right)=-b+O\left(\gamma^{-1}\right) \\
y \geq b,-d \leq z \leq d ; \\
E_{y 0} \approx-\frac{2 \gamma d b}{\pi y} \\
y>\gamma d \gg b,-d \leq z \leq d ; \\
E_{y 0} \approx-\frac{2 d b y}{\pi \gamma z^{2}} \\
z \gg d, \gamma d \gg b,-b \leq y \leq b ; \\
E_{z 0}=\frac{b}{\pi \gamma} \ln \frac{(d-z)}{(d+z)},|y-b|^{2} \ll \gamma^{2}|z-d|^{2} \\
E_{z 0}=-\frac{2 \gamma z d b}{\pi y^{2}},|y-b|^{2} \gg \gamma^{2}|z-d|^{2}, y>\gamma d \gg b
\end{gathered}
$$

For comparison,remember that for the point relativistic charge

$$
E_{y}=E_{\perp}=\frac{e}{R} \gamma, E_{z}=E_{\|}=\frac{e}{\gamma^{2} R}, R^{2}=x^{2}+y^{2}+\left(z-v_{0} t\right)^{2} ;
$$

i.e. the electric field for $\gamma \gg 1$ is practically transversal.In our case of the flat bunch expression (16-20) shows that always $\left|E_{z 0}\right| \ll\left|E_{y 0}\right|$, but the expressions for $E_{y 0}, E_{z 0}$ modified, compared to (21), due to the different geometry of the charge distribution. In what follows,we will use the approximate expressions for $E_{y 0}$ up to terms $\sim O\left(\gamma^{-1}\right)$, and put it to zero when $|z|>d$.

The next problem in zero approximation is the definition of the generalized velocities $V_{y 0}, V_{z 0}$. When $\gamma \gg 1$, and bunch length is short enough $d^{\prime} \ll 1,\left(d^{\prime}=k_{b} d\right)$ it is possible 
to drop out in (3) the terms proportional to $\left|V_{z 0}\right|^{2} \ll\left|V_{z 0}\right|$ and to $\left|V_{y 0}\right|^{2} \ll\left|V_{y 0}\right|$. Taking into account that in the considered case $\left|E_{y 0}\right| \gg\left|E_{z 0}\right|$ the equations of the motion (3) approximately can be rewritten in the following simple form

$$
\begin{array}{r}
\frac{\partial V_{z 0}}{\partial z} \approx V_{y 0} E_{y 0}, \\
\frac{\partial V_{y 0}}{\partial z} \approx\left(1+V_{z 0}\right) E_{y 0},|y| \leq b,|z| \leq d
\end{array}
$$

The solutions of eqs. (22) for the boundary condition $V_{y 0}=0, V_{z 0}=0$, when $z=d$ are:

$$
\begin{array}{r}
V_{y 0}=\sinh \left(\left|E_{y 0}\right|(d-z)\right) \simeq\left|E_{y 0}\right|(d-z), \\
V_{z 0}=\cosh \left(\left|E_{y 0}\right|(d-z)\right)-1 \simeq \frac{1}{2}\left|E_{y 0}\right|^{2}(d-z)^{2},
\end{array}
$$

where $E_{y 0}$ is given by (16-18) and $V_{y 0} \rightarrow 0, V_{z 0} \rightarrow 0$ when $y \rightarrow \pm \infty$. For the short $(d \ll 1)$ bunches from $(23)$ follows that $V_{z 0} \ll V_{y 0}$.

\section{FIRST ORDER APPROXIMATION}

Using eq. (11) in the first approximation (see (10)) and eq. (23) for $V_{y 0}$ it is possible to find $N_{1}(y, z)$ by the method of characteristics.

When $|y| \leq b, E_{y 0} \simeq-y+O\left(\gamma^{-1}\right)$ and characteristics are

$$
y e^{-\frac{1}{2}(d-z)^{2}}=C_{1}, y N_{1}^{(1)}=C_{2}
$$

The solution of eq. (24), which is equal to $\frac{n_{0}}{n_{b}}$, when $z=d$, is

$$
N_{1}^{(1)}=\frac{n_{0}}{n_{b}} e^{-\frac{1}{2}(d-z)^{2}}
$$

When $|y| \geq b, E_{y 0} \simeq-b+O\left(\gamma^{-1}\right)$ and characteristic is $-\frac{1}{2}(d-z)^{2}+\frac{y}{b}=C$, so $N_{1}^{(2)}$ is function of $-\frac{1}{2}(d-z)^{2}+\frac{y}{b}$; When $y=b$ it must coincides with the expression (25), so 


$$
N_{1}^{(2)}=\frac{n_{0}}{n_{b}} e^{-\frac{1}{2}(d-z)^{2}+\frac{y}{b}-1}
$$

When $y>\gamma d \gg b, E_{y 0} \simeq-\frac{2 b d \gamma}{\pi y}$ and characteristics are $y^{2}-\frac{2 b d \gamma}{\pi}(d-z)^{2}=C_{1}, \frac{N_{1}^{(3)}}{y}=C_{2}$ Taking into account that $N^{(3)} \rightarrow \frac{n_{0}}{n_{b}}$ when $|y| \rightarrow \infty$, we have

$$
N_{1}^{(3)}=\frac{n_{0}}{n_{b}} \frac{|y|}{\left[y^{2}-\frac{2 b d \gamma}{\pi}(d-z)^{2}\right]^{1 / 2}}
$$

Expressions (25-27) are the solutions of the eq. (24) for subsequent values of $V_{y 0}$ (23).Expression (26) for $z=d$ gives

$$
N_{1}^{(2)}=\frac{n_{0}}{n_{b}} e^{\frac{y}{b}-1}
$$

which seems as unappropriate. Taking into account, that (26) is an approximate expression,we assume the following interpolation formula for $N_{1}^{(2)}$ :

$$
N_{1}^{(2)}=\frac{n_{0}}{n_{b}} e^{-\frac{1}{2}(d-z)^{2}+c\left(\frac{y}{b}-1\right)(d-z)^{2}}
$$

which is $\frac{n_{0}}{n_{b}}$ when $z=d$, and coincides with the (25), when $y=b$.

$N_{1}^{(2)}$ is exponentially rising, when $y \rightarrow+\infty$ and at the some point $y_{c r}$ it intercept the $N_{2}^{(3)}$,which is also rising, when $y \rightarrow y_{p}+0 \equiv\left(\frac{2 b d \gamma}{\pi}\right)^{1 / 2}(d-z)<y_{c r}$. Hence $N_{1}$ has a maximum value and the plasma electrons distribution exibits a crest at the point $y=y_{c r}$.

Parameters $c$ and $y_{c r}$ may be defined by the condition

$$
N_{1}^{(2)}\left(y_{c r}, z\right)=N_{1}^{(3)}\left(y_{c r}, z\right)
$$

The second condition may be obtained,using the following consideration: longitudinal displacements of the plasma electrons are negligible,compared to transverse displacements, due to condition $V_{z 0} \ll V_{y 0}$,so for the given $|z| \leq d$ deficiency of the plasma electrons in the region $0<y<y_{c r}$ (and in the symmetric region $-y_{c r}<y<0$ ) is equal to the surplus of the plasma electrons in the region $y_{c r}<y<+\infty$ (and in the region $-\infty<y<-y_{\text {cr }}$ subsequently): 


$$
\begin{aligned}
& \int_{0}^{b}\left(\frac{n_{0}}{n_{b}}-N_{1}^{(1)}\right) d y+\int_{b}^{y_{c}}\left(\frac{n_{0}}{n_{b}}-N_{1}^{(2)}\right) d y= \\
= & \int_{y_{c}}^{y_{c r}}\left(\frac{n_{0}}{n_{b}}-N_{1}^{(2)}\right) d y+\int_{y_{c r}}^{\infty}\left(N_{1}^{(3)}-\frac{n_{0}}{n_{b}}\right) d y
\end{aligned}
$$

In (31) $y_{c}$ is the point, where $N_{\left(y_{c}, z\right)}^{(2)}=\frac{n_{0}}{n_{b}}$ From (30) for $d \ll 1$ :

$$
c=\frac{1}{2} \frac{\left(1+\frac{2 b d \gamma}{\pi y_{c r}^{2}}\right)}{\left(\frac{y_{c r}}{b}-1\right)} .
$$

From eq. (31) for $d \ll 1$ :

$$
y_{c r}=\frac{2 d \gamma}{\pi} \gg b
$$

and $c$ from (32) approximately has a following simple form:

$$
c \approx \frac{b}{2 y_{c r}}
$$

Now it is possible to turn to determination of the focusing force $W_{y 1}$ from eq. (14). For $\gamma \gg 1$ eq. (14) we get an approximate form

$$
\frac{\partial^{2} W_{y 1}}{\partial \bar{z}^{2}}+\frac{\partial^{2} W_{y 1}}{\partial y^{2}} \approx-\frac{\partial N_{1}}{\partial y}
$$

and using the Green function formalism the solution of eq. (35) can be written as

$$
W_{y 1}=-\frac{1}{2 \pi} \int d \bar{z}^{\prime} \int d y^{\prime} \frac{\partial N_{1}\left(y^{\prime}, \bar{z}^{\prime}\right)}{\partial y^{\prime}} \ln \left[\left(y-y^{\prime}\right)^{2}+\left(\bar{z}-\bar{z}^{\prime}\right)^{2}\right]^{1 / 2} .
$$

The domain of the integration in (36) is defined by the condition $\frac{\partial N_{1}(y, z)}{\partial y} \neq 0$,i.e. it is $-\infty<y<+\infty,-\infty<z \leq d$, because for $z>d, N_{1}=\frac{n_{0}}{n_{b}}$ and $\frac{\partial N_{1}}{\partial y}=0$.For $-d \leq z \leq d, \frac{\partial N_{1}}{\partial y}$ is given by (25),(27),(29);for $z<-d$ from the continuity condition $N_{1}(z, y)=N_{1}(-d, y)$ up to some $-z_{0}<-d$ where considered steady state regime changes to (nonlinear) wake wave (and later on the uniform distribution of the plasma electrons with $\left.N_{1}=\frac{n_{0}}{n_{b}}\right)$. 
Due to the symmetry of the Green-function and antisymmetry of the derivative $\frac{\partial N_{1}}{\partial y}$ it is possible to change the integration over the region $-\infty<y \leq 0$ to the integration over the region $0 \leq y \leq \infty$. Then the expression (36) has the following form:

$$
W_{y 1}=-\frac{1}{4 \pi} \int_{-\overline{z_{0}}}^{\bar{d}} d \bar{z}^{\prime} \int_{b}^{\infty} d y^{\prime} \frac{\partial N_{1}\left(y^{\prime}, \bar{z}^{\prime}\right)}{\partial y^{\prime}} \ln \frac{\left(y-y^{\prime}\right)^{2}+\left(\bar{z}-\bar{z}^{\prime}\right)^{2}}{\left(y+y^{\prime}\right)^{2}+\left(\bar{z}-\bar{z}^{\prime}\right)^{2}}
$$

taking into account (25). The interval of the integration over $y^{\prime}$ in (37) must be divided in the two intervals $\left(b, y_{c r}\right)$ and $\left(y_{c r}, \infty\right)$, where eq. (29) and (27) must be used for $\frac{\partial N_{1}}{\partial y}$; both expressions (27,29) have a maximum at $y^{\prime}=y_{c r}$ and it is possible approximately to take out of the integral the slowly variing logarithmic function at the maximum point $y^{\prime}=y_{c r}$. Then (37) will have the following form

$$
W_{y 1} \approx-\frac{1}{4 \pi} \int_{-\overline{z_{0}}}^{\bar{d}} d \bar{z}^{\prime} \ln \frac{\left(y_{c r}-y\right)^{2}+\left(\bar{z}-\bar{z}^{\prime}\right)^{2}}{\left(y_{c r}+y\right)^{2}+\left(\bar{z}-z^{\prime}\right)^{2}}\left[\frac{n_{0}}{n_{b}}-N_{1}^{(2)}\left(b, \bar{z}^{\prime}\right)\right]
$$

Notice that the approximate expression (38) for $W_{y 1}$ does not depend explicitely on the form of,then to some extent arbitrary,function $N_{1}^{(2)}$ (29), because $N_{1}^{(2)}(b, z)=N_{1}^{(1)}(z)$, see (29) and (25). Only the value of $y_{c r}(33)$, which enters in (38), explicitely depends on the form of $N_{1}^{(2)}$ through conditions (30, 31).

Taking into account, that $|y| \leq b \ll y_{c r}$ it is possible to develop the logarithm function under integral in a Taylor series, leaving the first term of the expression; then for $d \ll 1, y<$ $b$ the expression for $W_{y 1}$ will have the following form:

$$
W_{y 1}=\frac{1}{2 \pi} \frac{n_{0}}{n_{b}} y_{c r} \gamma y \int_{-z_{0}}^{d} d z^{\prime} \frac{\left(d-z^{\prime}\right)^{2}}{y_{c r}^{2}+\left(z-z^{\prime}\right)^{2} \gamma^{2}}
$$

Consider first the integral over $z^{\prime}$ in the domain $(-d, d)$, and later on in the domain $\left(-z_{0},-d\right)$.The resulting expressions denote by $W_{y 1}^{(0)}$ and $W_{y 1}^{(-)}$subsequently;then

$$
W_{y 1}^{(0)}=\frac{2 \alpha(z)}{\pi} \frac{n_{0}}{n_{b}} y d^{2}
$$

where $\alpha(z)$ is known function, which varies in interval $0,21 \leq \alpha(z) \leq 2,26$, when $-d \leq z \leq d, W_{y 1}^{(-)} \approx \frac{2}{\pi} \beta\left(z, z_{0}\right) \frac{n_{0}}{n_{b}} y d^{2}, \beta\left(z, z_{0}\right)$ weekly depends on the value of the $z_{0}$, and 
it is possible to take for estimates $z_{0} \rightarrow+\infty$. Then $\beta(z)=\beta(z, \infty)$ varies in interval $0,26 \leq \beta(z) \leq 1,57$, when $-d \leq z \leq d$.

Hence,for estimates,it is possible to use

$$
W_{y 1}=W_{y 1}^{(0)}+W_{y 1}^{(-)} \approx \frac{2}{\pi} y d^{2}[\alpha(z)+\beta(z)] \frac{n_{0}}{n_{b}} \approx \frac{4}{\pi} y d^{2} \alpha(z) \frac{n_{0}}{n_{b}}
$$

It is possible to apply the same approach to the flat,rigid,short positron bunch with uniform charge distribution.In this case in eqs. (15) electric field components change sign. As a consequence the $V_{0 y}$ component of the generalized velocity (eq. 23) also changes sign,which corresponds to attraction of the plasma electrons by positron bunch.Obviously enough,this leads to drastic change in plasma electrons redistribution as compared to electron bunch case.Instead of eq. (25) for $|y| \leq b$ it is possible to obtain

$$
N_{1 p}^{(1)}=\frac{n_{0}}{n_{b}} e^{\frac{1}{2}(d-z)^{2}}
$$

instead of (26) for $y \geq b$ we have

$$
N_{1 p}^{(2)}=\frac{n_{0}}{n_{b}} e^{\frac{1}{2}(d-z)^{2}+y / b-1}
$$

and instead of (27) for $y>\gamma d \gg b$

$$
N_{1 p}^{(3)}=\frac{n_{0}}{n_{b}} \frac{y}{\left[y^{2}+\frac{2 \gamma b d}{\pi}(d-z)^{2}\right]^{1 / 2}} ;
$$

From (42), (43) it is seen that plasma electrons concentrate around the rear part of the positron bunch.From (44) it is seen that $N_{1 p}^{(3)}$ has a complex pole and hence is slowly rising function,approaches it's limit equal $\frac{n_{0}}{n_{b}}$ from bellow, when $y \rightarrow+\infty$. This means that plasma electrons came to the region occupied by positron bunch, $|y| \leq b$,from the regions, where $y>\gamma d \gg b$.Focusing effect for the positron bunch case is caused also by plasma electrons redistribution.But due to different behaviour of $N_{1 p}^{(2)}$ and $N_{1 p}^{(3)}$, the interpolation between these two, which provide the main contribution to focusing force (12), (37), is much more arbitrary,than in the electron bunch case.In any choose of the interpolating function it must be more or less smooth,and therefore the estimates of the integrals like (36), (37) will be cumbersome enough. 
These two obstacles forced to think that positron bunch case must be treated numerically,even in the short bunch case and in the presented formalism.

\section{DISCUSSION}

The expressions for the focusing force,focusing gradient and stregth are subsequently

$$
f_{1 y}=-e W_{1 y}, G=\frac{W_{1 y}}{y}, K=\frac{e G}{\gamma m c^{2}},
$$

$W_{1 y}$ is given by (41), which is valid when $\gamma \gg 1$ and $d^{\prime} \ll 1\left(k_{b} d \ll 1\right)$.In the usual units expression (41) has the form

$$
W_{y 1}=\frac{64 \alpha(z) e r_{e}}{\pi}\left(n_{0} n_{b}\right)\left(y d^{2}\right)
$$

where $r_{e}=e^{2} / m c^{2}$ is the electron classical radius.Subsequently,focusing gradient and strength are:

$$
G=\frac{64 \alpha(z) e r_{e}}{\pi}\left(n_{b} n_{0}\right) d^{2}, K=\frac{64 \alpha(z) r_{e}^{2} d^{2}}{\gamma \pi}\left(n_{b} n_{0}\right)
$$

The expressions (47) differs from the previous results, which for the flat,long enough electron bunch and underdense lens are

$$
G=4 \pi n_{0} e, K=\frac{4 \pi r_{e} n_{0}}{\gamma}
$$

These are based on the understanding the focusing phenomenon as caused by the positive charge of the ion column,which exists inside and behind the moving electron bunch, because the considered bunch blows out the plasma electrons.

As it follows from the presented consideration (see,e.g.,eqs. (14, 35) the net focusing effect of the ion column,caused by noncompensated positiv charge $\frac{n_{0}}{n_{b}}-N_{1}\left(1+V_{z 0}\right)$, is proportional to $\gamma^{-2}$ and is negligible in our approximation $\gamma \gg 1$,compared to focusing effect of the plasma electrons redistribution,described by eqs. (35) and (39). The presence

of the factor $\gamma^{-2}$ in the part of the net (electric plus magnetic) focusing force,which caused 
by ions,is a result of magnetic compesation of the subsequent focusing electric field in lab frame, where electron bunch moves with the velocity $v_{0}$ and unperturbed parts of plasma are at rest. Indeed,from Maxwell eqs. (2.1), (2.4) in the first approximation:

$$
\begin{aligned}
\frac{\partial B_{1}}{\partial y} & =\beta N_{1} V_{z 0}+\beta \frac{\partial E_{z 1}}{\partial z}, \\
\frac{\partial E_{z 1}}{\partial z}+\frac{\partial E_{y 1}}{\partial y} & =\frac{n_{0}}{n_{b}}-N_{1}\left(1+V_{z 0}\right),
\end{aligned}
$$

it follows:

$$
\frac{\partial B_{1}}{\partial y}=-\beta\left[\frac{n_{0}}{n_{b}}-N_{1}\left(1+V_{z 0}\right)\right]+\beta N_{1} V_{z 0}-\beta \frac{\partial E_{y 1}}{\partial y},
$$

The first term in right hand side of eq. (50), being a part of the displacement current entered in (49),represents the nonuniform noncompensated positiv ions current,which propogate with the velocity $v_{0}$,equal to the velocity of the electron bunch.This term caused the compensating magnetic field,which at $\gamma \gg 1$ cancels the focusing action of the subsequent electric field.The physical reason for that - the exposed positiv ions column effectively "moves" in plasma along with the generating electron bunch with the velocity of it.When bunch,moving through plasma,blows out the plasma electrons,it uncower new forward parts of the partialy noncompensated ion column and this "motion" of the revealed positiv ions charge,i.e. subsequent current,generated compensating magnetic field.

The physical interpretation can be based also directly on the displacement current,entered in the first eq. (49) through the term $\beta \frac{\partial E_{z 1}}{\partial z_{1}}$. Ions ahead of the bunch are completely compensated by plasma electrons,inside the bunch ions charge is only partially compensated (eq. (25)), so exists the change of electric field component with $\tilde{z}=z-v_{0} t$, which generates subsequent compensating magnetic field.Formaly this part of displacement current coincides with the current conditioned by "effective ion motion", as it can be seen by replacing the electric field derivative in the first eq. (49) by it's value from Coulomb low-second eq. (49).It is evident that this coincidence is based on the steady state regime,adopted at the present work. 
In the bunch frame magnetic forces acting on the bunch electrons are absent.Electric field of the bunch charge has no relativistic contraction and hence the plasma electron redistribution has a different shape, more uniform, than in lab frame, which dimenish their focusing effect.But the focusing effect of the plasma electrons still is proportional to $n_{b}$-bunch charge density; their focusing electric field is relativistically contracted.These factors increase the redistributed plasma electrons focusing effect, which will be larger than focusing effect of the electric field of the ion column,proportional to $n_{0} \ll n_{b}$. Lorentz transformation did not changes the transverse component of the focusing force,so it must be the same in both frames.Above mentioned physical arguments allow to think that it could be the case.It is difficult to perform analytical calculations in the bunch frame due to complexity of relativistic equation of motion of the plasma electrons.Recall, that in presented calculations the plasma electrons considered as nonrelativistic, due to condition $\left(k_{b} d\right) \ll 1$.

All above mentioned general physical arguments are valid for the relativistic bunches with arbitrary length and charge distribution.Assumptions adopted in the present work (short enough relativistic bunch with the uniform charge distribution) allows us to perform analytical calculations.

Unfortunately the condition $k_{b} d \ll 1$ ( $d^{\prime} \ll 1$, short bunches) does not permit to use expression (47) for focusing strength for the flat beams of the FFTB [13]. In that case $n_{b}=7,7 \cdot 10^{18} \mathrm{~cm}^{-3}$ and $2,8 \cdot 10^{18} \mathrm{~cm}^{-3}$ and $k_{b}=5,2 \cdot 10^{5} \mathrm{~cm}^{-1}$ and $k_{b}=3,1 \cdot 10^{5} \mathrm{~cm}^{-1}$ consequently, $d=2,3 \cdot 10^{-2} \mathrm{~cm}$ and $k_{b} d=1,2 \cdot 10^{4} \gg 1$ and $0,7 \cdot 10^{4} \gg 1$.

Using the short bunches,nevertheless it is possible to obtain sufficiently strong focusing gradients.For example let $k_{b} d$ will be $k_{b} d=0,1$. Then $d=10^{-5}\left(\frac{3 \cdot 10^{19}}{n_{b}}\right)^{1 / 2}, G=7,5 \cdot 10^{-11} n_{0}$ and if $n_{0} \approx 10^{17} \mathrm{~cm}^{-3}$ as in FFTB case, $G \approx 7,5 \frac{\mathrm{MG}}{\mathrm{cm}}$.In order to fullfil the condition $\frac{n_{0}}{n_{b}} \ll 1, n_{b}$ must be e.g.,equal $10^{18} \mathrm{~cm}^{-3}$, then $d=5 \cdot 10^{-5} \mathrm{~cm}$. May be it is possible to obtain such a short bunches experimentally,using the technique,proposed in [19], [20].

It is worthwhile to stress, that due to specific form of the redistribution of the plasma electrons focusing gradient $\sim e^{3}$ and focusing strenght $\sim e^{4}$ have a quadrupole character, due to nonuniform shape of the distribution of plasma electrons along the crest,which 
diminish,inspite of the factor $\left(n_{0} n_{b}\right)$,significantly the numerical values of these quantities.

This disadvantage probably can be avoided,considering the medium $k_{p} d \geq 1$, or long $k_{p} d \gg 1$,bunches. The main difficulty in the analytic approach to these cases lies in the complex form of the equations of the motion (3- 5) which are fully relativistic in these cases.Probably the medium and long bunch cases can be treated by computational methods only.Presented analytical approximate description of the short bunch case can be used then as a physical guide and programs testing example.

It will be interesting to test experimentaly the predictions (47) for focusing gradient and strenght,particularly, the dependense of these quantities on $n_{b}$ and $d$.

\section{AKNOWLEDGEMENT}

Author would like to thank A.M. Sessler, who pointed out my attention on the plasma lens concept,for the careful reading preliminary versions of the manuscript,the numerous useful suggestions and criticism.Author is indebted to S.S. Elbakian,A.G. Khachatryan,J.S. Wurtele and S.G. Arutunian for attention, discussion and valuable comments.

I am obliged to G. Amatuni for the significant help in preparing the manuscript for publication. 


\section{References}

[1] P.Chen Part. Acc. 20,171,(1986)

[2] P.Chen,J.J. Su,T. Katzouleas,S. Wilks and J.M.Dawson IEEE Tran. Plasma Sci 15,218,(1987)

[3] P.Keinings,M.E.Jones Phys. Fluids 30,252,(1987)

[4] P.Chen,S.Rajagopalan and J.B.Rozenzweig Phys.Rev. D40,923, (1989)

[5] J.J.Su,T.Katzouleas,J.M.Dawson and R.F.Fedele Phys. Rev. A41, 3321,(1990)

[6] P.Chen,K.Oide,A.Sessler and S.Yu Phys. Rev. Lett. 64,1231, (1990)

[7] P.Chein Phys. Rev. Lett. A45,R3398,(1992)

[8] A.Ts.Amatuni,S.S.Elbakian,A.G.Khachatryan,E.V.Sekhpossian Part. Acc. (1995),in print,

Proc. $6^{\text {th }}$ Workshop on Adv.Acc. Concepts,pp 521-535, Fontana,1994;

AIP Conf. Proc.-335,ed P.Schoessow;

[9] J.B. Rozenzweig et all Phys. Rev. Lett. 61,98,(1988)

[10] J.B. Rozenzweig et all Phys. Fluids B2,1376,(1990)

[11] H.Nakanishi et all Phys.Rev. Lett. 61,98,(1991)

[12] G.Hairapetian et all Phys. Rev. Lett. 72,2403,(1994)

[13] W.Barletta et all,SLAC-PUB-95-6756,February 1995

[14] P.H.Whittum,A.M.Sessler,J.J.Stewart and S.S.Yu Part. Acc. 34,89,(1990).

[15] P.Chen In "The Physics of Beams", Proc. Andrew Sessler Simposium, p.68,Los Angeles,ed. W.A. Barletta,1993;AIP Conf. Proc.-351. 
[16] B.N.Breizman,T.Tajima,D.Fisher,P.Z.Chebotaev preprint Inst fur Fusion Studies Univ. of Texas at Austin,(1993)

[17] A.Ts. Amatuni,S.S.Elbakian,E.V.Sekhpossian preprint LBL-34836,UC-414,LBL,(1993)

[18] A.A.Sokolov,V.N.Ternov-"Relativistic electron","Nauka",M. 1974

[19] B.N.Breizman.P.Z.Chebotaev et all in Proc. $8^{\text {th }}$ Int. Conf. on High-Power Particle Beams (BEAMS-90),Novosibirsk,1990

[20] A.A. Bechtenev et all in Proc. XV Int. HEPACC,Hamburg,1992 